\title{
Antropología e innovación: Desarrollo comunitario desde la innovación culturalmente situada
}

\author{
Anthropology and innovation: Community development from culturally situated innovation
}

Norling Solís Narváez ${ }^{1}$

\section{Resumen}

La antropología ha constituido un campo relativamente longevo en nuestro continente; sin embargo, para Nicaragua es una ciencia reciente que ha avanzado sutilmente en las comunidades a través del acompañamiento desde el desarrollo comunitario. Las transformaciones socioeconómicas de Nicaragua, exigen re-estructuraciones económicas, sociales y culturales internas a las comunidades. Actualmente el campo de la innovación se ha vuelto un referente en la primera etapa de la juventud, en las comunidades, los barrios, los municipios, se fomentan las economías a través de innovaciones y por supuesto de emprendimientos, tanto de las instituciones públicas y privadas; empero, debemos hacer la pausa y preguntar ¿Son las innovaciones pertinentes culturalmente?, quizás sí, o quizás existen innovaciones que a través de los llamados influenciadores "influencers" llegan al público. En este ensayo se ha la reflexionado a la importancia de la etnografía aplicada a procesos de innovación en las comunidades, enfocados a fortalecer las capacidades locales, en función del reconocimiento de la cultura y necesidades focalizadas.

Palabras clave: Etnografía; innovación; cultura, metodología; antropología; economía.

\section{Abstract}

Anthropology has constituted a relatively long-lived field in our continent; however, for Nicaragua it is a recent science that has advanced subtly in the communities through accompaniment from community development. The socioeconomic transformations of Nicaragua require economic, social and cultural re-structuring internal to the communities. Currently the field of innovation has become a benchmark in the first stage of youth, in communities, neighborhoods, municipalities, fostering economies through innovations and of course of undertakings, both public and private institutions; However, we must pause and inquire: Are innovations culturally relevant?, perhaps yes, or perhaps there are innovations that come to the public through the so-called "influencers". In this essay, there is a reflection on the importance of ethnography

1 Doctor en Ciencias de la Educación,Profesor del Departamento de Antropología de la Universidad Autónoma de NicaraguaManagua. Correo: univernorling@gmail.com, su número Id: https://orcid.org/oooo-ooo1-9035-706X

Recibido: 05/02/2018 Aprobado: 30/04/2018 
applied to processes of innovation in communities, focused on strengthening local capacities, based on the recognition of culture and focused needs.

Keywords: Ethnography; innovation; culture; methodology; anthropology; economy.

\section{Introducción}

En el campo de las Ciencias Sociales la innovación parece un asunto complicado y difícil de aplicar, porque este componente está familiarizado en términos de tecnología,medicina o cualquier otro producto que sea utilizado por un mercado o sector poblacional. Las innovaciones generan un cambio concreto a través de un producto o servicio o bien satisfacen una necesidad inmediata para la población; sin embargo, cualquier innovación que representa un cambio o transformación para una población deberá ser analizada con cuidado. Es por ello que la etnografía es una metodología científica y útil para manejar el comportamiento de la población ante proyectos innovadores.

En este ensayo se aborda la importancia del uso de la etnografía para la innovación. El resultado del mismo surge de la puesta en práctica en la carrera de antropología del componente de la innovación y el análisis a proyectos que son innovadores pero que no funcionan por mucho tiempo.

La antropología, bajo el método etnográfico muestra técnicas de observación directa, observación participante y la entrevista estructurada, esto es fundamental para sacar todo el potencial a proyectos de innovación. El punto de partida de esta reflexión se encuentra en que nos centramos en lo tecnológico cuando se aborda el tema de innovación; pero lo importante ante cualquier proyecto es la perspectiva social, los sistemas de comportamiento cultural, la persona o grupo que será beneficiaria del proyecto innovador.

Evidentemente no existe innovación sin una necesidad y/o problemática a tratar. Cabe destacar que hemos de partir de un conocimiento cierto y sólido de las comunidades para las que desarrollamos la acción innovadora. Porque otra cosa que a veces se olvida es que no estamos innovando para segmentos, no ideamos soluciones para porcentajes poblacionales, sino para personas, que podemos agrupar, claro está, pero que hemos de entenderlas como tales y no como simples números.

\section{La etnografía: un acercamiento teórico y metodológico}

La antropología al igual que otras disciplinas tiene la intención de explicar fenómenos sociales a través del método etnográfico, que es distintivo de esta ciencia. La etnografía presenta un retrato de los más variados elementos de una cultura: temáticas 


\section{CIENCIAS SOCIALES}

como economía, organización social, sistema religioso, vinculación con la naturaleza y relación entre grupos sociales.

El método etnográfico se basa específicamente en la descripción detallada de los fenómenos sociales, esencialmente demuestra por medio de técnicas los puntos de vista del sector que está siendo investigado (Baztán, 1995). Además, el método cuenta con técnicas que permiten analizar en detalles un fenómeno o problemática. En palabras claves de Ángel Baztán, la etnografía es "el estudio descriptivo de la cultura de una comunidad o de alguno de sus aspectos fundamentales bajo la perspectiva de comprensión global de la misma" (Baztán, 1995, pág. 5).

Por tanto, se infiere directamente como la forma holística de describir la cultura de una comunidad o contexto de estudio, perpetrando en la idea de entrar al campo y realizar una fotografía descriptiva y analítica de la comunidad. Descriptiva, porque refleja un panorama de realidad en estudio, y analítica porque supone un análisis profundo de por qué ocurre de esa manera.

Rossana Guber, una antropóloga que dedicó una obra al análisis profundo del quehacer reflexivo de la etnografía, hace referencia a puntos centrales de los cuales ocuparemos para aterrizar la idea de una innovación aplicada, real y concreta.

Etnografía es, en primer lugar, un argumento acerca de un problema teórico-social y cultural suscitado en torno a cómo es para los nativos [población local] de [121] una aldea, una villa miseria, un laboratorio o una base espacial, vivir y pensar del modo en que lo hacen. Los elementos del texto etnográfico son 1) La pregunta o problema, 2) La respuesta, explicación, o interpretación, 3) Los datos que incluye como evidencias para formular el problema y para darle respuesta, y 4) La organización de estos elementos (problema, interpretación y evidencia) en una secuencia argumental (Jacobson, 1991:2) y (Guber, 2001, pág. 127).

En esta definición del autor compromete a los antropólogos o cientista social a realizar una investigación basada en la observación, descripción e interpretación del contexto y sujetos de estudio. Implica una tarea amplia, en tal razón se adjudica a la antropología como una ciencia holística. La etnografía trata precisamente de contextualizar los discursos y las prácticas de los actores, tomando en cuenta el contexto real de interacción social entre los sujetos locales. No es únicamente observar por observar, sino ser un actor más en el contexto que se estudia, dando pautas a ser observador como observado.

En la actualidad los estudios con el método etnográfico conciben una forma más enriquecida de investigar dando vida a todos los elementos que componen la realidad de una comunidad, desde la percepción del juego de miradas (Velásquez, 2001). 
Las grandes obras, innovaciones y proyectos de cualquier tipo requieren un proceso integral para descifrar y dar respuesta real a una necesidad, apoyándose de una técnica de investigación sustancial: la observación. Aunque desde el enfoque antropológico, Bronislaw Malinowski sugirió el participar, lo que se denomina:

la observación participante (que) se caracteriza por la existencia de un conocimiento previo entre ambos y una permisibilidad en el intercambio establecido, lo cual da lugar a una iniciativa por parte de cada uno de ellos en su interrelación con el otro (Argigalga, 1989).

La consistencia de la investigación etnográfica es tratar de verificar la obtención de los datos de campo, así que la "La observación participante consiste en dos actividades principales: observar sistemática y controladamente todo lo que acontece en torno del investigador, y participar en una o varias actividades de la población. Hablamos de "participar" en el sentido de "desempeñarse como lo hacen"; de aprender a realizar ciertas actividades y a comportarse como uno más. La "participación" pone el énfasis en la experiencia vivida por el investigador apuntando su objetivo a "estar adentro" de la sociedad estudiada." (Guber, 2001, pág. 57).

Por tanto, al participar, o más bien al observar cómo se interrelacionan los elementos de la cultura local, hay mayores posibilidades de atender una necesidad, puesto que las respuesta reales y concretas las tienen los mismos pobladores o sector meta. La observación también se analiza bajo un criterio de necesidad para la investigación antropológica, mencionando que "la observación directa era posible dar fe de distintos aspectos de la vida social desde una óptica no etnocéntrica, superando las teorías hipotéticas evolucionistas y difusionistas del siglo XIX" (Guber, 2001, 59).

En ese devenir de la investigación etnográfica, se debe relacionar lo que se observa con la entrevista. Básicamente "La entrevista es una estrategia para hacer que la gente hable sobre lo que sabe, piensa y cree (...), una situación en la cual una persona (el investigador entrevistador) obtiene información sobre algo interrogando a otra persona" (Guber, 2001, pág. 75).

La entrevista, o la conversación con la población no es sólo levantar el dato, es descifrar la necesidad/problemática que tiene una interpretación o connotación particular desde los involucrados locales, a fin de cuenta son ellos los que serán afectados por cualquier proyecto que llegue.

Es importante desarrollar la visión antropológica en los estudios de proyectos de innovación, para dar respuesta a las interrogantes acerca de la realidad de los individuos desde adentro. Es importante señalar que la antropología como ciencia ha 


\section{CIENCIAS SOCIALES}

logrado escudriñar la cultura de una comunidad desde adentro. Desarrollar una mirada antropológica para observar e interpretar que es la idea principal de la etnografía.

El método etnográfico comprende dos enfoques de observación: Emic y Etic. Los investigadores sobre todo antropólogos toman posición desde el Etic, ya que describe los fenómenos observados desde el punto de vista del observador, básicamente pretende describir comportamientos y hacer una interpretación en base a esas observaciones. El enfoque Emic busca interpretar las acciones y comportamiento desde el punto de vista del investigado, definiéndolo por criterios subjetivos.

Lo que arroja reales resultados más allá de cumplimientos de objetivos en ambas perspectivas es la subjetividad a veces negada por algunos investigadores, o bien promotores de proyectos. La subjetividad es una realidad misma de las localidades, no sólo supone ese "ser interior", sino más bien una estructura social y culturalmente compleja, la cual debe ser contemplada en cualquier estudio para que tenga sentido para los beneficiarios o actores locales.

Puesto que la cultura misma que no es más que una creación humana y como tal es reinventada con perpetuos procesos dialécticos y desarrollados por procesos subjetivos humanos. Propiamente dicho la cultura niega en sí misma la existencia de "parámetros objetivos de carácter racional" para juzgar una sociedad o un tipo de práctica social en relación a otra (Rey, 2013).

Tradicionalmente, el objetivo de la observación participante ha sido detectar las situaciones en que se expresan y generan los universos culturales y sociales en su compleja articulación y variedad. La aplicación de esta técnica, o mejor dicho, conceptualizar actividades tan disímiles como «una técnica» para obtener información supone que la presencia (la percepción y experiencia directas) ante los hechos de la vida cotidiana de la población garantiza la confiabilidad de los datos recogidos y el aprendizaje de los sentidos que subyacen a dichas actividades (Guber, 2001).

La experiencia que va generando subjetividad en los actores locales son quizás la fuente de conocimiento más rica para el investigador etnógrafo y en todo caso para el promotor de proyectos de innovación, sociales y demás.

La representación ideal de la observación es tomar notas de una obra de teatro como mero espectador. Desde el ángulo de la observación, entonces, el investigador está siempre alerta pues, incluso aunque participe, lo hace con el fin de observar y registrar los distintos momentos y eventos de la vida social (Guber, 2001).

La intención básica de toda investigación etnográfica es naturalista, es decir, trata de comprender las realidades actuales, entidades sociales y percepciones humanas, así 
como existen y se presentan en sí mismas, sin intrusión alguna o contaminación de medidas formales o problemas preconcebidos. Es un proceso dirigido hacia el descubrimiento de muchas historias y relatos idiosincrásicos, pero importantes, contados por personas reales, sobre eventos reales, en forma real y natural (Miguelez, 2005).

\section{La innovación culturalmente para el desarrollo comunitario}

La innovación es una explotación exitosa -viable y real- de nuevas ideas con una clara orientación al cliente o beneficiario. Por su connotación es asumida en el mundo empresarial como una función única para aquellas organizaciones que podían permitírselo. Sin embargo, hoy día constituye un factor clave de competitividad dado el nuevo contexto socioeconómico ( Fundación EDE, 2009).

La definición general expuesta en el Manual de Oslo, compartida por la OCDE y EUROSTAT, señala que las innovaciones:

comprenden los nuevos productos y procesos, así como las modificaciones tecnológicas importantes de los mismos. Una innovación en el ámbito económico se considera como tal cuando es introducida en el mercado, es decir se crean productos claves y quizás o a veces ni muy necesarios. Y también existen innovaciones en procesos, organizaciones o administraciones utilizado lógicamente para reducir costes o mejorar la calidad.

La teoría de la innovación, por tanto, ha manejado tradicionalmente dos tipos de innovación: la de producto y la de proceso -ya sea externa (nuevos servicios añadidos al producto) o interna (organizativa). Obviamente las empresas, organizaciones no lucrativas y administraciones públicas que pertenecen o actúan en el sector servicios también innovan en sus "productos-servicios" (los que prestan y ofrecen a sus clientes) y en sus procesos actividades internas y/o externas que los hacen más eficientes y eficaces ( Fundación EDE, 2009).

Por otro lado, además del impacto local, también puede hablarse de cierto dinamismo global. Manuel Castells (1999), hace referencia al concepto globalización, entendida como la articulación entre lo global y lo local, como una noción que hoy se aplica tanto a la economía como a la cultura. La globalización supone destacar el ámbito urbano y el papel gestor-coordinador, promotor de los gobiernos locales para la implantación de políticas que tienen en cuenta unos referentes globales y que se posicionan respecto a ellos.

Por lo tanto, supone que las innovaciones van en paralelo a la globalización, o al desarrollo; sin embargo, puede que no sea así. Si posicionamos la innovación desde un enfoque global y no aterrizado a la realidad local, expone una creciente vulnerabilidad 


\section{CIENCIAS SOCIALES}

de la comunidad afectada. Para la CEPAL abordar el tema de la innovación social gira en torno a la mayoría de las innovaciones del campo social como readaptaciones creativas en procesos y contextos distintos a los originales.

De ahí el valor que agrega la diseminación de las ideas al crecimiento de las innovaciones, bajo el supuesto de que ellas surgen y se replican en situaciones con características particulares. Aunque el propósito de una innovación sea resolver una necesidad particular de un grupo específico, lo cierto es que en su mayoría tienen origen en las soluciones alcanzadas por otro grupo en otro contexto, y su destino muy posiblemente sea seguir aplicándose en una escala superior, para beneficio de un mayor número de personas.

En su réplica y su multiplicación, que la innovación alcanza su pleno sentido, pues la lucha contra la exclusión será plenamente exitosa cuando ninguna persona esté excluida; por ello, aunque el éxito de una innovación particular no dependa de su réplica, esta le proporciona un sentido social (Herrera \& Alvarado Ugarte , 2008). Siguiendo un patrón de réplicas existen proyectos que pueden ser adaptados y exitosos en distintos puntos, por ejemplo, la adaptación del modelo educativo cubano "Yo sí puedo", en Nicaragua, eso demuestra que existen innovaciones que se pueden bien replicar, con obvia pertinencia cultural.

En efecto, como sugieren Herrera y Alvarado (2008), la réplica de una innovación pareciera dar sentido colectivo, pero replicar la experiencia innovadora, aun cuando ha sido viable en distintos lugares, debe contener previamente un acercamiento etnográfico desde la ubicación cultural, que le dé en principio sentido de pertenencia y pertinencia a la innovación donde se desarrollará.

La visión etnográfica colabora con brindar un conocimiento integral del que será un potencial usuario o beneficiario, y no sólo como miembro de un segmento económico o mercado que adquiera un producto o servicio determinado, sino cómo un actor social parte de un colectivo. Entre los elementos que destacan tener un proyecto con visión etnográfica es estar inmerso en el contexto que brindará de por sí un criterio fuera del parcialismo de lo malo y bueno. Todas las costumbres internas en una comunidad tienen una lógica de ser, por tanto, los resultados no pueden arrojar que el proyecto no funciona porque cierto comportamiento de la población es "malo" o "inadecuado". De esa manera, se podría decir que funcionan réplicas de proyectos situados. Cuando al diagnóstico previo de un proyecto se ante ponen criterios evaluadores de la anterior experiencia, ese puede ser un principio de fracaso.

El proyecto apoyado de un buen estudio etnográfico ha de aplicar el relativismo cultural a sus observaciones, afirmando que la validación de proyecto se vincule a la interpretación que hace el investigador de la realidad y por supuesto la de los sujetos estudiados. Por supuesto al desarrollar un proyecto de innovación se intenta 
interiorizar las necesidades reales de los sujetos estudiados o comunidad, no las que el agente externo sustente en sus creencias externas.

\section{Reflexiones finales}

Finalmente, la etnografía es una herramienta científico metodológica que es útil no sólo para ejecutar investigaciones como tal, sino para realizar diagnósticos de proyectos innovadores, o bien proyectos sociales, que sean aplicados a ciertos grupos con particularidades culturales.

Por tanto, se convierte en una estrategia de conocimiento que genera a través de un etnógrafo levantamiento de datos confiables, basados en la observación y la conversación. Ambas técnicas que requieren sobre todo un proceso eminente libre de sesgos, para que pueda arrojar datos puntualmente sustanciales.

La aplicación correcta de las técnicas y por supuesto del vivir cultural permite que las apreciaciones de los actores locales sean convincentes para el proyecto planteado. Por tanto, no se omite las visiones culturales de los sujetos en estudio a cualquier caso particular o proyecto en específico, por supuesto esto genera adaptaciones, o configuraciones adecuadas y pertinentes antes de su ejecución completa.

Entre otros puntos, es importante ver al segmento poblacional, y beneficiario no como futuro consumidor o agente económico del proyecto, sino como actor social, que puede validar la innovación y colectivizarla entre sus grupos cercanos. Esto supone que compartirá su apreciación con otros actores partes de otros grupos y eso potencializa el proyecto. Pero debe cumplir con el criterio culturalmente situada, y por tanto que responde a necesidades mediadas entre quien dirigió el proyecto y quienes validaron el mismo.

Con lo anterior se sustenta que no es suficiente comprender que realiza o hace un grupo, sino que se vuelve sustancial analizar por qué lo hace, esto genera conocimiento sustancial para ejecutar una innovación. Además, si fuese el caso de réplica, una misma innovación, no resulta exactamente igualmente aceptada en otro espacio sino tiene previamente el estudio etnográfico para situarlo culturalmente.

Por tanto la etnografía permite tener esa visión holística que se necesita para tener conocimiento del grupo social donde se prevé ejecutar proyectos sociales o innovadores, estos se sitúan en una comunidad, una empresa, un mercado o una población (Sobejano, 2014). Aunque no implica que estén lejos de pertenecer a segmentos sociales, por tanto, vinculan su desarrollo a estructuras sociales complejas que requieren análisis pertinente. 
Principalmente como han sugerido antropólogos clásicos como Maurice Godelier, Stuart Plattner, entre otros, lo económico es tan sustancial como parte de la cultura en un grupo social. Por tanto, para comprender como responden económicamente a ciertos fenómenos o elementos deben vincular todos los aspectos de su vida social, como parte de su cotidianidad. En eficacia a ello, se logrará tener un grupo receptivo a la innovación diseñada, puesto que ya se comprendió su entorno social, cultural, y económico.

\section{Lista de referencias}

Argigalga, M. T. (1989). Metodología de la observación en las ciencias humanas. Madrid: Cátedra.

Baztán, A. A. (1995). Etnografia: Metodlogía de Investigación Cualitativa. Dialnet, 1-25.

Fundación EDE. (20 de Febrero de 2009). Seminario sobre Innovación Social en el Ámbito de los Servicios Sociales. Obtenido de http://www.fundacionede.org/innovacion/ docs/contenidos_innovacion/seminario_innovacion_conclusiones.pdf

Guber, R. (2001). La Etnografia. Metodo, Campo y Reflexividad. Bogota: Norma.

Guber, R. (2001). La etnografía: método, campo y reflexividad. Barcelona: Grupo Editorial Norma.

Herrera, A. R., \& Alvarado Ugarte , H. (2008). Claves de la innovación en América Latina y el Caribe. Chile: CEPAL .

Miguelez, M. M. (12 de Diciembre de 2005). Plataforma Lecturas. Obtenido de http:// prof.usb.ve/miguelm/metodoetnografico.html

Rey, F. L. (2013). Subjetividad, cultura e investigación cualitativa en psicología: la ciencia como producción culturalmente situada. LIMINALES. Escritos sobre psicología y sociedad / Universidad Central de Chile, 13-36.

Sobejano, J. (2014). Metodologías etnográficas para el desarrollo de innovaciones consistentes. Sintetia.

Velásquez, J. R. (2001). El trabajo etnográfico. Un olvido de la antropología física. Estudios de Antropología Biológica, 635-653. 